ment that in the Theorie Analytique des Probabilités (Livre II., chap. ii., \$ 4) a closely approximate formula is given for their easy calculation. No doubt the process by which this formula is obtained is somewhat difficult as well as troublesome, but the existence of the formula itself should be generally known.

When it is applied to the ahove problem, it gives the answer in the somewhat startling form of "ahout 40,000 years."

P.S.-April 4, 1887.-Finding that Laplace's formula ceases to give approximate results, for very large values of $m$ and $n$ when these numbers are of the same order of magnitude, $I$ applied to Prof. Cayley on the subject. He has supplied the requisite modifcation of the formula, and his paper has been to-night communicated to the Ruyal Society of Edinburgh.

Sur les cordes communes à une conique et a un cercle de rayon nul;

Application a la thóorie géométrique des foyers dans les coniques.

Par M. Maurice d'Ocagne.

1. Etant donnés une conique $K$, dont l'équation est $K=0$, et t.n point $\mathbf{P}(\alpha, \beta)$, l'equation générale des coniques qui passent par les points d'intersection de la conique $K$ et da cercle $P$ de rayon nul, qui a le point $(\alpha, \beta)$ pour centre, est

$$
\mathrm{K}+\lambda\left[(x-a)^{2}+(y-\beta)^{2}\right]=0 .
$$

Comme les quatro points dintersection du cercle $P$ et de la conique $K$ sont imaginaires, le système (1) comprend un seul couple de droites réelles $\Delta$ et $\Delta$. Ces droites seront dites, par analogie avec une expression proposée par Chasles, * les conjointes du point $P$ et de la conique $K$.

Parmi les couples de cordes irnaginaires communes an cercle $P$ et a la conique $\mathrm{K}$ se trouvent les droitse isotropes passant au point $\mathbf{P}$

$$
\begin{aligned}
& (x-a)+i(y-\beta)=0 \\
& (x-\alpha)-i(y-\beta)=0,
\end{aligned}
$$

\footnotetext{
* Journal de Lioniville, T. III., p. 385.
} 
c'est-a-dire, les droites qui unissent lo point $P$ aux ombilics $I$ ot $J$ du plan (expression de Laguerre), points imaginaires situés sur la droite de l'infini et par où passent tous les cercles du plan.

Une première oonséquence de cette remarque est que le point $P$ a méme polaive relativement à la conique $K$ et aux conjointes $\triangle$ et $\Delta^{\prime}$. Les conjointes passent en effet, d'uprès ce qui précède, par les points communs a la conique $K$ et aux droites PI et PJ.

Par conséquent, le point de rencontre des conjointes $\Delta$ et $\Delta^{\prime}$ se trouve sur la polaire du point $P$ relativement a la conique $K$. On peut observer, aussi, qu'en vertu d'une propriété générale des coniques passant par l'intersection d'une conique et d'un cercle, les conjointes $\Delta$ et $\Delta^{\prime}$ sont également inclinées sur los axes de la conique $\boldsymbol{K}$.

Une deuxième conséquence de la remarque faite plus haut est que parmi les conjointes d'un point et d'un cercle se trouve toujours la droite a l'infini du plan. L'autre conjointe, situé \& distanre finie, est laxe radioal du point et du cercle, dont les propriétés sont bien connues.

O^ voit tout de suite que les conjointes du centre d'une ellipse

$$
x^{2} / a^{2}+y^{2} / b^{2}=1 \quad(n>b)
$$

et de cette courbe sont données par

$$
y= \pm a b / c
$$

et celles du centre d'une hyperbole

$$
x^{2} / a^{2}-y^{2} / b^{2}=1
$$

par

$$
x= \pm a b / c
$$

Dans le cas de l'ellipse, les tangentes menées des extrémités du grand axe au cercle qui a pour diamètre le petit axe, coupent ce petit axe aux points par ò̀ passent les conjointes du centre, qui sont, daillewrs, paralleles au grand axe. C'est la traduction de la formule

$$
y= \pm a b / c \text {. }
$$

2. L'importance de la considération des conjointes réside dans le théorème suivant:-

Dans la transformation par polaires réciproques relativement à un cercle, les éléments corrélatifs des foyers d'une conique sont les conjointes du centre du cercle directeur (centre de la transformation) et do la conique corrélative.

La démonstration de ce théorème est des plus simples. Las ombilics $I$ et $J$ étant situés sur la droite de l'infini, et les directions 
isotropes $O I$ et $O J$ ( $O$ est le centre de la transformation) étant rectangulaires, les ombilics $I$ et $J$ ont respectivernent pour éléments corrélatifs les droites $O J$ at $O r$. $O r$, les foyers d'une conique $K$ sont les points de rencontre réels des couples de tangentes menées à $K$ par les ombilics $I$ et. $J$. Les éléments corrélatifs de ces foyers seront donc les cordes communes à la conique corrélative de $\mathrm{K}$ et au couple de droites $O J$ et $O I$, c'est- $\$$-dire, les conjointes du centre $O$ de la transformation, et de la conique corrélative de $\mathrm{K}$.

A titre de corollaire immédiat de ce théorème on peut remarquer que si l'un des foyers de la conique $K$ coïncide avec le centre $O$ de la transformation, l'une des conjointes du point $O$ et de la conique corrélative étant rejetée à l'infini, cette conique correllative est un cercle, résultat bien connu dont on pénètre ainsi la raison intime.

Le théorème précédent permettra de transiormer les propriétés des foyers des coniques en propriétés de conjointes, et vice versd. En particulier, on pourra considérer les systèmes de coniques ayant les mêmes cordes réelles communes avec un point donné, coniques qui pourront être dites homoconjonctives par rapport à ce point, et toutes leurs propriétés se déduiront corrélativement des proprićtés bien connues des systèmes de coniques homofocales.

Mais ici nous nous attacherons surtout-c'est là le principal objet de ce petit Mémoire-a faire voir comment le théorème précédent peut être utilisé pour déduire les propriétés focales des coniques des propriétés tout élémentaires de l'axe radicai d'un cercle et d'un point.

3. Soient $C$ un cercle, $O$ un point, $f$ l'axe radical de ce cercle et de ce point (droite équidistante du point $O$ et de la polaire de ce point relativement au cercle $\mathrm{C}$ ).* Une transformation par polaires réciproques de centre $\mathrm{O}$ donne comme courbe corrélative du cercle $\mathrm{C}$ une conique $\mathrm{K}$, qui est une ellipse, une hyperbole ou une parabole, selon que le point $O$ est a l'intérieur, a l'extérieur ou sur la circonférence du cercle $C$. Mais, dans tous les cas, cette conique $K$ a pour foyers le point $O$ et le point $F$ corrélatif de la droite $f$.

4. Joignons le point $O$ à un point $M$ pris sur le cercle $C$; la tangente en $M$ au cercle $C$ coupe la droite $f$ au point $N$; tirons $O N$ et menous $\mathrm{O} \mu$ parallelement à $\mathrm{MN}$.

* Lo lecteur est prí́ de faire les figures. 
D'après une propriété fondamentale de l'axe radical $f$ du point $O$ et du cercle $\mathrm{C}$, on a $\mathrm{NO}=\mathrm{NM}$;

$$
\text { donc } \angle \mathrm{NOM}=\angle \mathrm{NMO} \text {, et } \angle \mathrm{NOM}=\angle \mathrm{MO} \mu^{*}{ }^{*}
$$

L'élément corrélatif du point $M$ pris sur le cercle $C$ est une tanyente $m$ à la conique $\mathrm{K}$; celui de la droite $f$ est le foyer $F$ de la conique $K$, l'autre foyer átant au point $O$; celui de la tangente $M N$ au cercle $\mathrm{C}$ est le point de contact $\mathbf{P}$ de la tangente $m$ sur la conique $K$; ceux des points $N$ et $\mu$ (ce dernier situé a l'infini dans la direction MN) sont les rayons vecteurs PF et PO. La transformation do la propriété précédente montre donc que l'angle de $\mathrm{PF}$ avec la tangente $m$ est égal à l'sngle de $m$ avec PO. On obtient ainsi cette propriété classique :-

La tangente en un point d'une conique est bissectrice de l'angle des rayons veeteurs qui unissent le point de contact aux foyers de la coniqu.

5. Les polaires d'un point $M$, pris sur l'axe radical de deux cercles, relativement à ces deux cercles, se coupent sur leur axe radical, car elles sont elles-mêmes les axes radicaux du cercle de centre $M$ orthogonal aux cercles donnés et de ces cercles. Lorsque l'un des cercles donnés se réduit à un point $O$ cette propriété derient : si la polaire, relativement a un cercle $C$ d'un point $M$ pris sur l'axe radical d'un point $O$ et de ce cercle $O$ coupe cet axe radical au point $M^{\prime}$, l'angle MOM' est droit. Transformant par polaires réciproques, on a ce théorème bien connu :-

Le pôle d'une droite passant par le foyer $F$ d'une conique, relativement à cette conique est sur la perpendiculaire élevée en $F$ à cette droitc.

Ou bien :-

La perpendiculaire meriée par un foyer $F$ d'une conique au rayon vecteur d'un point $M$ de cette conique coupe la tangente en $M$ sur la directrice relative au foyer $k$. $\dagger$

- Une transformation homographiquo permet de deduire de là le théo. rème suivant:-Les segmients d'une tangente à une conique, compris entre le point de contact de cette tangcnte et ses intersections avec les conjointes d'un point par rapport à ha conique, sont vus de ce point sous des angles égaus.

$\dagger$ On pout aussi remarquer, on observent que l'blément corrélatif du centre d'an cercle $\mathbf{C}$ ent la direstrice de la conique corrélative $\mathbf{K}$ qui corres. pond an foyer confonda aveo le centre $O$ do la transformation, que cette pro. priété est également corrélative de celle-ci :-

T'outes les normales a un cerele passent par le centre de ce cercle. 
6. Tout cercle dont le centre $M$ est sur la droite $f$ el qui passe par le point $O$ coupe orthogonalement le cercle $C$, c'est-ì-dire qu'il passe par les points de contact des tarigentes menées de $M$ au cercle C. La transformation par polaires réciproques de cette propriété montre que si $F$ et $F^{\prime}$ sont les foyers d'une conique $K$ et que $M^{\prime} M^{\prime}$ soit une corde de la conique passant par le foyer $\mathbf{F}$, la parabole qui a $F^{\prime}$ pour foyer et $\mathrm{MM}^{\prime}$ pour directrice ost tangente aux tangentes menées d la conique $\mathrm{K}$ par les points $M$ et $\mathbf{M}^{\prime}$. En outre, si $\mu$ et $\mu^{\prime}$ sont les points de contact de ces tangentes et de la parabole les angles $M F^{\prime} \mu$ et $M^{\prime} F^{\prime} \mu^{\prime}$ sont droits. Donc, d'après lo théorème qui tormine le No. 5 , les points $\mu$ et $\mu^{\prime}$ appartiennent a la directrice de la conique $K$ relative au fojer $\mathrm{F}^{*}$. On est ainsi conduit a ce théorème qui ne nous semble pas avoir étó dójà remarqué.

Cns parabole qui a pour foyer un foyer $F^{\prime} d^{\top}$ une conique $K$ et pour dirsctrice une droite quelconque passant par l'autve foyer $F$ de la coniqus $K$ at coupant cette oonique aux points $M$ st $M^{\prime}$, est tangents aux tangentes à la conique $K$ menés par les points $M$ et $M$, la corde de contact étant la directrice de la conique $\boldsymbol{K}$ relative "u foyer $F^{\prime}$.

7. Soient $f$ et $f_{1}$ les axes radicaux d'un point $O$ et de deux cercles $O$ et $C$. La perpendiculaire abaissée du point de rencontre $\operatorname{de} f$ et $f_{1}$ sur la ligne des centres de $\mathrm{C}$ et $\mathrm{C}_{1}$ est l'axe radical de ces deux cercles. La transformation par polaires réciproques, le point $O$ étant toujours pris pour centre de la transformation, donne ce théorène qui nous semble également nouveau :-

Si deux coniques ont en commun un foyer $O$, ces coniques ont un pôle doublo réel qui est d la rencontre de la droite qui joint leo autreg foyers $F$ et $F_{1}$ et do la perpendiculaire élevée en $O \dot{O}$ la droite qui joint ce foyer au point de rencontre des directrices qui lui correspondent dans les deux coniquies.

8. Si $t$ et $t^{\prime}$ sont les tangentes menóes d'un point $M$ ḋ un cercle $G$, et que $D$ soit un point pris sur la polaire du point $M$ relativement au cercle $O$, la polaire $d$ du point $D$ passe par le point $M$, et les droites $d$ ec MD sont conjuguées harmoniques par rapport aux droites $t$ et $t^{\prime}$.

Supposons alors que le point $M$ se trouve sur l'axe radical $f d$ u point $O$ et du cercle $O$, et que nous prenions pour point $D$ le point de la polaire de $M$ qui se trouve sur la polaire de $O$; la droite $d$ se confond alors avec MO. Soient ' $\mathrm{T}$ et $\mathrm{T}^{\prime}$ les points de contact avec $\mathrm{C}$ 
des tangentes issues de $\mathbf{M}$; puisque $\mathbf{M T}=\mathbf{M T} \mathbf{T}^{\prime}=\mathbf{M O}$, si par le point $O$ nous élevons à $O{ }^{\prime} T$ et a $O T^{\prime \prime}$ les perpendiculaires $O N$ et $O N^{\prime}$ qui coupent $M T$ et $M T^{\prime}$ respectivenent en $N$ et en $N^{\prime}$, nous avons $T N=2 T M$, $T^{\prime} N^{\prime}=2 T^{\prime} M$; par suite, la droite $N^{\prime} N^{\prime}$ est symétrique de $T^{\prime} T^{\prime}$ par rapport a $\mathrm{M}$, ot si cette droite coupo $\mathrm{MD}$ en $E, O E$ est parallele a $f$.

Cela posé, opérons une transformation par polaires réciproques de centre $O$. Au point $M$ situé sur $f$ correspond une droite passant par le foyer $F$ de la conique $K$ et conpant cette conique en deux points $A$ et $A^{\prime}$. Anx points $N$ et $N^{\prime}$ correspondent les normaies a la conique $K$ en $A$ at $A$, nornales qui se coupent en $B$. Au point $E$ correspond la parallèle à l'axo focal de la conique $K$, menée par le point $B$; si cette parallele coupe $A_{A}{ }^{\prime}$ au point $C$, le point $O$ est dè lors corrélatif de la droite $M E$; mais nous venons de voir que la droite $M D^{\prime}$ est conjuguée harmonique de MO par rapport aux tangentes MT et $\mathrm{MT}^{\prime}$; donc le point $\mathrm{O}$ est conjugué harmonique du point situé a l'infini sur $A A^{\prime}$ par rapport aux points $A$ ot $A^{\prime}$, e'est-dindire que le point $M$ est le milieu de $\mathbf{A A}^{\prime}$, et nous obtenons ce thérème connu :--

$S i$ par le point de rencontre des normales à une conique menés par les extrémités d'une corde focale, on mène une parallele à l'axe focal de cette conique, cette droite passe par le milieu de la corde focale considérée.

9. Supposant toujours le point $M$ situé sur l'axe radical $f$ du point $O$ et du cercle $\mathrm{C}$, plaçons, maintenant, le point $\mathrm{D}$ a la rencontre de la polairo du point $M$ relativement au cerclo $C$ et de la droite qui joint le centre de ce cercle au point O-c'est-a-dire, au póle de la droite $f$. La droite $d$ coincide alors avec la droite $f$, et nous royons que MD est conjuguee harmonique de $f$ par rapport aux tangentes au cercle $C$ issues de $M$, ou, si ces tangentes coupent en $H$ et en $H^{\prime}$ la parallèle a $f$ menée par $\mathrm{D}$, que $\mathrm{D}$ est le milieu de $\mathrm{HH}^{\prime}$, et, par suite, que les angles HOD et DOH' sont egaux.

Transformons toujours par polaires réciproques :

Au pole $\mathrm{D}$ de la droite $f$ correspond la directrice de la conique $\mathrm{K}$ relative au foyer $\mathrm{F}$. On a donc ce théoreme connu :

Si la corde $A A^{\prime}$ d'une conique passe par le foyer $F$ de cette conique, les droites, qui joignent les extrémités $A$ et $A^{\prime}$ de cette corde au point de rencontre do la directrice retative aus foyer $F$ ' st de l'axe focal, sont également inclinées sur cet axe.

10. Considerons un cercle $\mathrm{C}$ de centre $\Omega$ (fig. 82 ), un point $\mathrm{O}$ et 
une droite $l$ quelconque perpendiculaire à $O \Omega$. Joignons lo point $O$ a un point $P$ mobile sur le cercle $C$; menons $O Q$ perpendiculaire a OP et $Q R$ parallèle à la tangente $P T$ menée en $P$ au cercle $C$, c'estis-dire perpendiculaire à Po. Nous avons

$$
\angle \mathrm{OQR}=\angle \mathrm{OP \Omega}=0, \angle \mathrm{OQH}=\angle \mathrm{PO} \Omega=\phi .
$$

Donc, si nous abaissons du point $O$ sur $Q R$ la perpendiculaire $O R$, nous avons

$$
\mathrm{OR}=O Q \sin \theta=\mathrm{OH}_{\mathrm{H}} \frac{\sin \theta}{\sin \phi}=\mathrm{OH}_{-} \frac{\mathrm{O}_{2}}{\mathrm{P} \Omega},
$$

et comme $\mathrm{OH}, \mathrm{O} \Omega$ et $\mathrm{P}_{\Omega}$ sunt constants, $\mathrm{OR}$ est aussi constant; par suite, la drnite $Q R$ enveloppe un cercle de centre $O$.

Transformons par polaires réciproques. Nous avons une conique $K$ ayant un foyer au point $O$. Au poixt $P$ correspond une tangente $t$ a cette conique; a la droite $d$, un point $\triangle$ de laxe focal ; au point $Q$, la pexpendioulaire $p$ absissée de $\Delta$ sur $t ; a$ la droite $Q R$ qui joint le point $Q$ au point situé a l'infini sur la tangente $P$ 'T au cercle $C$, le point de rencontre de la droite $p$ et du vecteur qui joint le foyer $O$ au point de contact de la tangente $t$ et de la conique $\mathbf{K}$. D'ailleurs le cercle de centre $O$ enveloppé par $Q R$ a pour corrélatif également un cercle de centre $O$. On a donc ce théorème, connu :-

Le lieu du point de rencontre du royon vecteur qui unit un point $M$ mobile sur une conique à l'un des foyers de cette conique et de la perpendiculaire abaissée d'un point $\Delta$ de l'caxe focal sur la tangente au point $M$, est un cercle ayjant pour centre le foyer $O$.

Si on prend pour point $\triangle$ le second foyer $F$ de la conique, on voit, en considérant le point $M$ dans une position infiniment voigine de l'un des sommets do l'axe focal, que le rayon du cercle correspondant (cercle directeur) est égal a la longueur de cet axe. Rapprochant ce résultat du théorème obtenu au No. 4, on en déduit la propriété des rayons vecteurs dans les coniques.

11. Nous donnerons encore un exenple remarquable de la métbode que nous indiquons ici.

Supposons que le cercle $\mathbf{C}$ soit variable, mais ait constamment avec le point $O$ même axe radical $f$. Considérons en outre un point fixe quelconque $D$. L'axe radical des points $D$ et $O$ considérés comme cercles de rayon nul est la perpendiculaire élovée a DO en son milieu. Cette droite coupe $f$ en un point $K$, et l'axe radical du point $D$ et du cercle $C$ passe constamment par le point $K$. 
11 en résulte que la polaire du point $D$ relativement an cercle $C$ passe constamment par le point $D^{\prime}$ symétrique du point $D$ par rapport au point $\mathrm{K}$, point situé à la rencontre de $\mathrm{DK}$ et de la perpendiculaire élevéo en $\mathrm{O}$ a $O D$.

Passons à la figure corrélative. Aux différents cercles $\mathrm{C}$ correspondent des coniques ayant toutes un foyer au point $O$ et un foyer au point $F$, c'est-a-dire des coniques homofocales, et si $d$ est la droite corrélative du point $D$, nous voyons que les poles de cette droite par rapport aux coniques du système sont situés sur une droite $d$, la corrélative du point $D^{\prime}$. Le point $K$ a pour élément corrélatif la droite qui joint le point $F$ au symétrique du point $O$ par rapport $\grave{k}$ la droite $d$. Cette droite coupe la droite $d$ en un point $M$, et puisque l'angle DOD' est droit, la droite $d$ est la perpendiculaire élevéo en $\mathrm{M}$ is la droite $d$. De ld ce théorème connu :-

Les pôles d'une droite $d$ relativement $\grave{a}$ un systìme de coniques homofocales sont situés sur la perpendiculaire menée à cette droite par le point ò̀ elle est coupée par la droite qui joint l'un des foyers au symétrique de l'autre par rapport d̀ $d$.

Remarquant que la droite $d$ est tangente au point $M$ a une conique ayant pour foyers $F$ et $O$, or peut énoncer encore ce théorème de la manière que voici.

Le lieu des pôles d'une droite d relativement à un système de coniques homofocales est la normale à celle de ces coniques qui touche la droile $d$, menée par le point de contact de cette conique et de cette droite.

12. Nous nous hornerons aux exemples qui précèdent pour mettre en relief la fécondité de la méthode qui consiste à déduire les propriétés focales des coniques de la théorie des axes radicaux.

Pour terminer, nous ferons observer que réciproquement toute propriété des foyers conduit corrélativeunent à une propriété des conjointes d'un point et d'une conique, et, plus particulierement, de l'axe radical d'un point et d'un cercle.

Exemple.-Prenons cette propriété connue : Soit MFM' une corde focale de l'ellipse dont le grand axe est $\mathrm{AA}^{\prime}$; si l'on prolonge $\mathrm{MA}$ et $M^{\prime} A$ jusqu'd leurs points de rencontre $Q$ et $Q$ avec la directrice qui correspond au foyer $\mathrm{F}$, l'angle $\mathrm{QF} \mathrm{Q}^{\prime}$ est droit.*

- Rouché et de Comberousse, Traité de Géométrie, T. II., $5^{\circ}$ \&dit., p. 529, Eix. 916. 
Appelens $O$ le second foyer de la conique, et transhurmons par polaires réciproques en prenant le point $O$ pour centre de la transformation. Nous obtenons ainsi ce théorème :-

Soient $f$ l'axe radical l'un point $O$ et d'un cercle $C$, et $P$ le pôle de cet axe relativement au cercle $C$. Si les tangentes mentes d'un point quelconque de $f$ ars cercle $C$ coupent l'une des tangentes $\grave{a}$ co cercle parallèles à $f$ aux points $I$ et $T$, et que les droites $P I$ et $P I$ coupent la droite $f$ aus points $H$ et $H^{\prime}$, l'angle $H O H$ est droit.

\section{Note on the Kinematics of a Quadrilateral.}

\section{By R. J. Dalilas.}

I send a note on the following problem, a solution of which was requested of me by one of the tutors at King's College, Cambridge.

We are given a quadrilateral of four jointed bars ABCD (fig. 83). The bar CD being held fast, find the tangent to the locus of $P$, the intersection of DA, CB in any position ; and verify the following construction for the radius of curvature of the path of $P$ :-

Let $P Q$ be the third diagonal, draw through $P$ a perpendicular to $P Q$ meeting $B A, C D$ in $L$ and $L^{\prime}$; through $L$ and $L^{\prime}$ draw parallels to $P Q$ meeting $A D$ in $M$ and $M^{\prime}$; through $M$ and $M^{\prime}$ draw perpendiculars to $\mathrm{AD}$ meeting the normal at $\mathrm{P}$ in $\mathrm{O}$ and $\mathrm{O}^{\prime}$; then will

$$
-1 / \rho=1 / O P+2 / O^{\prime} P \text {. }
$$

The first part of this is easily found. The important angles in the figare have been marked thus-

$$
\mathrm{CPQ}=\alpha, \mathrm{DPQ}=\beta, \mathrm{AQP}=\gamma, \mathrm{DQP}=\epsilon .
$$

Making $P D$ rock through a small angle $\delta \theta$, we have, if $\delta$ is the resulting element of arc traced out by $P$,

$$
\begin{aligned}
& \delta s=\mathrm{PD} \delta \theta \operatorname{cosec} T \mathrm{PD}, \text { and so also, if } \phi=\angle \mathrm{PCD}, \\
& \delta s=\mathrm{PC} \delta \phi \operatorname{cosecTPC} .
\end{aligned}
$$

Now $\delta \phi / \delta \theta=Q D / Q C$ as is well known, (see Goodeve's Elements of Mechanism p. 110), and thus $\frac{\sin \mathrm{TPC}}{\sin \mathrm{T} \cdot \mathrm{PD}}=\frac{\mathrm{QD}}{\mathrm{PD}} \frac{\mathrm{PC}}{\mathrm{QC}}$.

Now

$$
\frac{Q D}{P D}=\frac{\sin P P Q}{\sin P Q D} \text { and } \frac{P C}{Q C}=\frac{\sin P Q O}{\sin Q P \bar{C}}
$$

therefore

$$
\frac{\sin \mathrm{TPC}}{\sin \mathrm{TPD}}=\frac{\sin \beta}{\sin \alpha} \text { and } \mathrm{TPC}-\mathrm{TPD}=\beta-\alpha ;
$$

therefore

$$
\mathrm{TPC}=\beta \text { and } \mathrm{TPD}=\alpha \text {. }
$$

